\title{
Predictors of New Dementia Diagnoses in Elderly Individuals: A Retrospective Cohort Study Based on Prefecture-Wide Claims Data in Japan
}

\author{
Yuriko Nakaoku ${ }^{1,2, *}$, Yoshimitsu Takahashi ${ }^{1}$, Shinjiro Tominari ${ }^{1}$ and Takeo Nakayama ${ }^{1}$ \\ 1 Department of Health Informatics, Kyoto University School of Public Health, Kyoto 606-8501, Japan; \\ takahashi.yoshimitsu.3m@kyoto-u.ac.jp (Y.T.); tominari-s@umin.ac.jp (S.T.); \\ nakayama.takeo.4a@kyoto-u.ac.jp (T.N.) \\ 2 Department of Preventive Medicine and Epidemiology, National Cerebral and Cardiovascular Center, \\ Suita 564-8565, Japan \\ * Correspondence: yurikon@kuhp.kyoto-u.ac.jp; Tel.: +81-6-6170-1070
}

check for updates

Citation: Nakaoku, Y.; Takahashi, Y.; Tominari, S.; Nakayama, T. Predictors of New Dementia Diagnoses in Elderly Individuals: A Retrospective Cohort Study Based on Prefecture-Wide Claims Data in Japan Int. J. Environ. Res. Public Health 2021, 18, 629. https://doi.org/10.3390/ ijerph18020629

Received: 22 December 2020 Accepted: 9 January 2021 Published: 13 January 2021

Publisher's Note: MDPI stays neutral with regard to jurisdictional clai$\mathrm{ms}$ in published maps and institutional affiliations.

Copyright: (C) 2021 by the authors. Licensee MDPI, Basel, Switzerland. This article is an open access article distributed under the terms and conditions of the Creative Commons Attribution (CC BY) license (https:// creativecommons.org/licenses/by/ $4.0 /)$.

\begin{abstract}
Preventing dementia in elderly individuals is an important public health challenge. While early identification and modification of predictors are crucial, predictors of dementia based on routinely collected healthcare data are not fully understood. We aimed to examine potential predictors of dementia diagnosis using routinely collected claims data. In this retrospective cohort study, claims data from fiscal years 2012 (baseline) and 2016 (follow-up), recorded in an administrative claims database of the medical care system for the elderly (75 years or older) in Niigata prefecture, Japan, were used. Data on baseline characteristics including age, sex, diagnosis, and prescriptions were collected, and the relationship between subsequent new diagnoses of dementia and potential predictors was examined using multivariable logistic regression models. A total of 226,738 people without a diagnosis of dementia at baseline were followed. Of these, 26,092 incident dementia cases were detected during the study period. After adjusting for confounding factors, cerebrovascular disease (odds ratio, 1.15; 95\% confidence interval, 1.11-1.18), depression (1.38; 1.31-1.44), antipsychotic use $(1.40 ; 1.31-1.49)$, and hypnotic use $(1.17 ; 1.11-1.24)$ were significantly associated with subsequent diagnosis of dementia. Analyses of routinely collected claims data revealed neuropsychiatric symptoms including depression, antipsychotic use, hypnotic use, and cerebrovascular disease to be predictors of new dementia diagnoses.
\end{abstract}

Keywords: claims data; dementia; medical care system for the elderly; predictors; prescription

\section{Introduction}

Dementia among elderly people is an important public health concern. The number of elderly people living with dementia exceeded 50 million worldwide in 2018, and is projected to triple by 2050 [1]. In Japan, the world fastest aging society, those aged $\geq 65$ years and $\geq 75$ years accounted for $27 \%$ and $13 \%$ of the population, respectively, in 2015 [2]. The Hisayama study, which comprised volunteer Japanese residents, reported that the prevalence of dementia among individuals aged $\geq 65$ years increased from 1985 to 2012 for all-cause dementia (from 7\% to 11\%) and Alzheimer's disease (from 2\% to 7\%) [3].

Previous studies have identified some predictors of dementia [4-6]. One meta-analysis has identified seven potentially modifiable predictors for dementia: diabetes mellitus, hypertension, obesity, smoking, physical inactivity, cognitive inactivity, and depression [7]. In 2020, the Lancet commission report has identified 12 modifiable risk factors for dementia: less education, hypertension, hearing impairment, smoking, obesity, depression, physical inactivity, diabetes, infrequent social contact, excessive alcohol consumption, head injury, and air pollution [6]. Several risk prediction models have been developed based on demographics, neuropsychological testing scores, and magnetic resonance imaging data [8]. 
However, these studies examined selected volunteers as participants and used variables that are not routinely collected in medical care. Consequently, the applicability of prediction models for real practices, as well as estimation of entire population burden, are somewhat limited [9].

Administrative claims data cover usual medical care for all insured people. Although these data are potentially useful for examining dementia predictors, previous studies on this topic have been limited. Accordingly, this study aimed to examine the usefulness of routinely obtained administrative claims data for identifying predicters of new dementia diagnoses in a Japanese elderly population.

\section{Materials and Methods}

\subsection{Design and Data Source}

This was a retrospective cohort study. In 2008, Japan initiated the medical care system for the elderly aged $\geq 75$ years. We extracted data from an administrative claims database covering almost all residents aged $\geq 75$ years in Niigata prefecture. Enrollment in this insurance system is mandatory for all Japanese citizens except for welfare recipients. We obtained anonymized claims data for fiscal year (FY) 2012 (baseline) and FY2016 (followup), which included inpatient/outpatient medical diagnoses (coded by the International Classification of Diseases 10th revision, ICD-10) and prescription drugs (categorized according to the Anatomical Therapeutic Chemical classification system, ATC code). For each person, data were merged using a unique but anonymous identification code. The follow-up period was 60 months, from April 2012 to March 2017, according to the Japanese fiscal year which starts on 1 April and ends on 31 March.

This study was approved by the ethics committee of the Kyoto University Graduate School and Faculty of Medicine (No. R1767). Participant written consent was waived because this study involved routinely collected claims data that were de-identified before provision and anonymously managed at all stages, including data cleaning and statistical analysis.

\subsection{Study Population}

Figure 1 shows a flowchart of participant selection. We selected participants from the data source according to the following criteria: (1) those residing in Niigata prefecture who were insured by the medical care system for the elderly aged $\geq 75$ years from FY2012 to FY2016, and (2) those with no record of dementia diagnosis in FY2012. We could not obtain claims data for individuals covered by public assistance, so these individuals were not included in the study.



Figure 1. Flowchart of participant selection. 


\subsection{Exposure Including Predictors}

We selected variables which could be obtained from the administrative claims database as potential risk factors of dementia $[5,6,10]$. The following predictor variables were included in the risk model:

1. Demographic measures: age, sex.

2. Medical diagnosis: diabetes mellitus (ICD-10: E11-E14), ischemic heart disease (I20-I25), cerebrovascular disease (I60-I69), atrial fibrillation/flutter (I48) at baseline, and current depression diagnosis (F32, F33)/treatment with antidepressants.

3. Prescriptions: antihypertensive drugs (ATC-code: C02, C03, C07, C08, C09), antihyperlipidemia drugs (C10), antidepressants (N06A), antipsychotics (N05A), anxiolytics (N05B), hypnotics (N05C), and antithrombotics (B01AC).

Prevalent users were defined as those who had been prescribed any of the abovementioned drugs at least once at baseline. According to Walter's definition of predictors for dementia and availability in our database, we defined exposure variables as follows: treated with antihypertensive drugs, dyslipidemia drugs, hypnotics, antipsychotics, or anxiolytics, and received depression diagnosis/treatment with antidepressants [10].

\subsection{Outcomes}

The outcome was a new diagnosis of dementia at follow-up (FY 2016), which was determined from the following medical diagnoses coded by the ICD-10: dementia in Alzheimer's disease (F00), vascular dementia (F01), dementia in other diseases (F02), unspecified dementia (F03), Alzheimer's disease (G30), and other degenerative diseases of the nervous system (G31). Hessler's criteria were used for dementia diagnoses [11]. To increase the validity of the diagnoses, dementia cases were narrowed to participants who received diagnoses in at least two billing quarters of health insurance or both in- and outpatient settings, as was done in a previous analysis of claims data [11].

\subsection{Statistical Analysis}

For the descriptive analysis, continuous variables were reported as means and standard deviations (SD), while categorical variables were reported as numbers and percentages. To examine potential predictors of a new diagnosis of all-cause dementia, we used multivariable logistic regression models. One model was adjusted for age and sex (ageand sex-adjusted model). Another model was adjusted for age, sex, and potential risk factors for dementia (medical diagnosis and/or prescriptions), including comorbidities of cerebrovascular disease, diabetes mellitus, ischemic heart disease, atrial fibrillation, and depression or use of antidepressants, and use of antipsychotics, anxiolytics, hypnotics, antihypertensive drugs, dyslipidemia drugs, and antithrombotics. In order to elucidate the aging effect of these potential predictors, we also analyzed the data by age group: $75-79$ years, $80-84$ years, and $\geq 85$ years at baseline. In addition, the effects of potential predictors on sub-types of dementia (Alzheimer's disease and vascular dementia) were also examined.

Analyses were performed using Stata 15.0 software (StataCorp, College Station, TX, USA). All reported $p$ values were two-tailed, and the threshold for significance was $p<0.05$.

\section{Results}

\subsection{Demographic and Clinical Characteristics of the Study Cohort}

We identified 341,910 people eligible for the medical care system at entry. Of these, 54,819 people with codes for dementia at baseline were excluded. We also excluded 60,353 people who died or moved out within 5 years of the observation period, for analytical purposes (Figure 1). Ultimately, data from 226,738 people were analyzed (baseline characteristics are shown in Table 1). Mean age was 80.0 years in men and 81.3 years in women. Males were more likely to have a history of cerebrovascular disease, diabetes mellitus, ischemic heart disease, and atrial fibrillation, compared to females. Females were more 
likely than males to have a history of hypertension and dyslipidemia, and to be prescribed antidepressants, antipsychotics, anxiolytics, and hypnotics.

Table 1. Baseline characteristics of the study cohort by sex.

\begin{tabular}{|c|c|c|c|c|c|c|}
\hline \multirow[t]{2}{*}{ Characteristics } & \multicolumn{2}{|c|}{ Total } & \multicolumn{2}{|c|}{ Male } & \multicolumn{2}{|c|}{ Female } \\
\hline & \multicolumn{2}{|c|}{$n=226,738$} & \multicolumn{2}{|c|}{$n=82,145$} & \multicolumn{2}{|c|}{$n=144,593$} \\
\hline $\begin{array}{l}\text { Baseline age, mean (SD) } \\
\text { Comorbidities, } \mathrm{n}(\%)\end{array}$ & 80.81 & $(4.67)$ & 80.02 & $(4.18)$ & 81.26 & $(4.87)$ \\
\hline Cerebrovascular disease & 61,979 & $(27.3 \%)$ & 22,889 & $(27.9 \%)$ & 39,090 & $(27.0 \%)$ \\
\hline Diabetes mellitus & 63,406 & $(28.0 \%)$ & 26,202 & $(31.9 \%)$ & 37,204 & $(25.7 \%)$ \\
\hline Ischemic heart disease & 37,955 & $(16.7 \%)$ & 14,838 & $(18.1 \%)$ & 23,117 & $(16.0 \%)$ \\
\hline Atrial fibrillation & 17,520 & $(7.7 \%)$ & 8866 & $(10.8 \%)$ & 8654 & $(6.0 \%)$ \\
\hline Depression & 16,845 & $(7.4 \%)$ & 3828 & $(4.7 \%)$ & 13,017 & $(9.0 \%)$ \\
\hline Hypertension & 154,132 & $(68.0 \%)$ & 54,477 & $(66.3 \%)$ & 99,655 & $(68.9 \%)$ \\
\hline Dyslipidemia & 98,723 & $(43.5 \%)$ & 28,848 & $(35.1 \%)$ & 69,875 & $(48.3 \%)$ \\
\hline \multicolumn{7}{|l|}{ Prescriptions, n (\%) } \\
\hline Antidepressants & 10,272 & $(4.5 \%)$ & 2248 & $(2.7 \%)$ & 8024 & $(5.5 \%)$ \\
\hline Antipsychotics & 7344 & $(3.2 \%)$ & 2084 & $(2.5 \%)$ & 5260 & $(3.6 \%)$ \\
\hline Anxiolytics & 53,707 & $(23.7 \%)$ & 13,195 & $(16.1 \%)$ & 40,512 & $(28.0 \%)$ \\
\hline Hypnotics & 18,704 & $(8.2 \%)$ & 4428 & $(5.4 \%)$ & 14,276 & $(9.9 \%)$ \\
\hline Antihypertensive drugs & 148,663 & $(65.6 \%)$ & 52,575 & $(64.0 \%)$ & 96,088 & $(66.5 \%)$ \\
\hline Dyslipidemia drugs & 68,662 & $(30.3 \%)$ & 17,735 & $(21.6 \%)$ & 50,927 & $(35.2 \%)$ \\
\hline
\end{tabular}

Abbreviation: SD, standard deviation.

\subsection{Characteristics of Incident Dementia Cases}

We identified 26,092 people who were diagnosed with dementia during the study period; 19,093 were classified as Alzheimer's disease (ICD-10: F00, G30), 604 as vascular dementia (F01), and 5875 as unspecified dementia (F03) (Table 2). The overall prescription prevalence of anti-dementia drugs was $63 \%$, and prevalence by age showed a decreasing trend with increasing age. Prescriptions of anti-dementia agents also differed by type of dementia, with those with Alzheimer's disease having the highest rate of prescriptions (78\%), and rates of anticholinesterase and memantine prescriptions of $67 \%$ and $19 \%$, respectively (Table 2).

Table 2. Characteristics of incident dementia cases.

\begin{tabular}{ccccc}
\hline Type of Dementia & Alzheimer's Disease & Vascular Dementia & Unspecified Dementia & All-Cause Dementia \\
\hline Dementia incidence, $\mathrm{n}$ & 19,093 & 604 & 5875 & 26,092 \\
Age, $\mathrm{n}(\%)$ & & & \\
75-79 y & $6591(34.5 \%)$ & $201(33.3 \%)$ & $1603(27.3 \%)$ & $8627(33.1 \%)$ \\
$80-84 \mathrm{y}$ & $7447(39.0 \%)$ & $211(34.9 \%)$ & $1990(33.9 \%)$ & $9835(37.7 \%)$ \\
$\geq 85 \mathrm{y}$ & $5055(26.5 \%)$ & $192(31.8 \%)$ & $2282(38.8 \%)$ & $7630(29.2 \%)$ \\
Prescriptions, $\mathrm{n}(\%)$ & & & & \\
Any anti-dementia drugs & $14,912(78.1 \%)$ & $128(21.2 \%)$ & $1110(18.9 \%)$ & $16,330(62.6 \%)$ \\
Anticholinesterases & $12,851(67.3 \%)$ & $98(16.2 \%)$ & $886(15.1 \%)$ & $14,006(53.7 \%)$ \\
Memantine & $3670(19.2 \%)$ & $41(6.8 \%)$ & $318(5.4 \%)$ & $4050(15.5 \%)$ \\
\hline
\end{tabular}

\subsection{Logistic Regression Analysis}

Table 3 shows the results of multivariable logistic regression analyses of predictors of a new diagnosis of dementia. Cerebrovascular disease (odds ratio (OR), 1.15; 95\% confidence interval (CI), 1.11-1.18), depression/antidepressant use (OR, 1.38; 95\% CI, 1.31-1.44), antipsychotic use (OR, 1.40; 95\% CI, 1.31-1.49), hypnotic use (OR, 1.17; 95\% CI, 1.11-1.24), and antithrombotic use (OR, 1.06; 95\% CI, 1.02-1.10) were positive predictors of a new diagnosis of dementia, whereas antihypertensive drug use (OR, 0.90; 95\% CI, 0.88-0.93) and dyslipidemia drug use (OR, $0.92 ; 95 \%$ CI, $0.89-0.95)$ were negative predictors of dementia in the multivariable-adjusted model. Multivariable adjustments for possible confounders 
only marginally altered the results of the age- and sex-adjusted model. Additional analysis revealed that the above-mentioned items were also predictors of a new diagnosis of Alzheimer's disease (Supplementary Materials, Table S1), while cerebrovascular disease (OR, 2.20; 95\% CI, 1.83-2.65), depression/antidepressant use (OR, 1.33; 95\% CI, 1.02-1.74), and antipsychotic use (OR, 2.25; 95\% CI, 1.64-3.07) were identified as positive predictors of a new diagnosis of vascular dementia (Supplementary Materials, Table S2).

Table 3. Multivariable logistic regression analyses of predictors for new diagnosis of dementia.

\begin{tabular}{|c|c|c|c|c|}
\hline \multirow[t]{2}{*}{ Predictors } & \multicolumn{2}{|c|}{ Age- and Sex- Adjusted Model } & \multicolumn{2}{|c|}{ Multivariable-Adjusted Model ${ }^{a}$} \\
\hline & OR $(95 \% C I)$ & $p$-value & OR $(95 \% C I)$ & $p$-value \\
\hline Age (per 1-year increase) & $1.06(1.06-1.07)^{b}$ & $<0.001$ & $1.06(1.06-1.07)$ & $<0.001$ \\
\hline Female & $1.19(1.16-1.22)^{\mathrm{C}}$ & $<0.001$ & $1.18(1.14-1.21)$ & $<0.001$ \\
\hline Cerebrovascular disease & $1.19(1.16-1.23)$ & $<0.001$ & $1.15(1.11-1.18)$ & $<0.001$ \\
\hline Diabetes mellitus & $1.02(0.99-1.05)$ & 0.152 & $1.03(1.00-1.06)$ & 0.075 \\
\hline Ischemic heart disease & $1.00(0.97-1.04)$ & 0.857 & $0.99(0.95-1.02)$ & 0.430 \\
\hline Atrial fibrillation & $0.98(0.93-1.02)$ & 0.321 & $0.97(0.92-1.02)$ & 0.233 \\
\hline Depression or use of antidepressants at baseline & $1.56(1.50-1.63)$ & $<0.001$ & $1.38(1.31-1.44)$ & $<0.001$ \\
\hline Use of antipsychotics at baseline & $1.71(1.61-1.82)$ & $<0.001$ & $1.40(1.31-1.49)$ & $<0.001$ \\
\hline Use of anxiolytics at baseline & $1.15(1.11-1.18)$ & $<0.001$ & $1.00(0.96-1.03)$ & 0.851 \\
\hline Use of hypnotics at baseline & $1.33(1.27-1.38)$ & $<0.001$ & $1.17(1.11-1.24)$ & $<0.001$ \\
\hline Use of antihypertensive drugs at baseline & $0.94(0.91-0.97)$ & $<0.001$ & $0.90(0.88-0.93)$ & $<0.001$ \\
\hline Use of dyslipidemia drugs at baseline & $0.94(0.91-0.96)$ & $<0.001$ & $0.92(0.89-0.95)$ & $<0.001$ \\
\hline Use of antithrombotics at baseline & $1.12(1.09-1.15)$ & $<0.001$ & $1.06(1.02-1.10)$ & 0.001 \\
\hline
\end{tabular}

Abbreviations: OR, odds ratio; CI, confidence interval. a Adjusted for age, sex, cardiovascular risk factors, and neurological or psychiatric risk factors. ${ }^{\mathrm{b}}$ The OR of age was adjusted for sex. ${ }^{\mathrm{c}}$ The OR of sex was adjusted for age.

The age-group analysis $(75-79,80-84$, and $\geq 85$ years) revealed that the predictive ability of cerebrovascular disease, diabetes mellitus, and use of psychiatric medications for a new diagnosis of dementia decreases with age (Table 4). 
Table 4. Multivariable logistic regression analysis of predictors for new diagnosis of dementia by age group.

\begin{tabular}{|c|c|c|c|}
\hline & $75-79 y$ & $80-84$ y & $\geq 85 y$ \\
\hline Predictors & OR $(95 \% \mathrm{CI})$ & OR $(95 \% \mathrm{CI})$ & OR $(95 \%$ CI) \\
\hline Age (per 1-year increase) & $1.14(1.12-1.16)$ & $1.08(1.06-1.10)$ & $0.99(0.99-1.00)$ \\
\hline Female & $1.19(1.14-1.25)$ & $1.23(1.17-1.29)$ & $1.12(1.06-1.19)$ \\
\hline Cerebrovascular disease & $1.25(1.18-1.32)$ & $1.13(1.08-1.19)$ & $1.06(1.00-1.13)$ \\
\hline Diabetes mellitus & $1.12(1.06-1.17)$ & $0.98(0.93-1.03)$ & $0.97(0.91-1.03)$ \\
\hline Ischemic heart disease & $0.99(0.92-1.05)$ & $1.01(0.95-1.07)$ & $0.96(0.90-1.03)$ \\
\hline Atrial fibrillation & $1.06(0.97-1.16)$ & $0.95(0.87-1.02)$ & $0.87(0.79-0.96)$ \\
\hline $\begin{array}{c}\text { Depression or use of antidepressants at } \\
\text { baseline }\end{array}$ & $1.47(1.36-1.59)$ & $1.38(1.28-1.49)$ & $1.21(1.10-1.32)$ \\
\hline $\begin{array}{l}\text { Use of antipsychotics } \\
\text { at baseline }\end{array}$ & $1.48(1.33-1.64)$ & $1.40(1.25-1.56)$ & $1.31(1.15-1.49)$ \\
\hline Use of anxiolytics at baseline & $1.03(0.97-1.10)$ & $0.95(0.89-1.01)$ & $0.97(0.91-1.05)$ \\
\hline Use of hypnotics at baseline & 1.17 (1.07-1.28) & $1.19(1.10-1.30)$ & $1.16(1.04-1.29)$ \\
\hline $\begin{array}{l}\text { Use of antihypertensive drugs } \\
\text { at baseline }\end{array}$ & $0.89(0.85-0.94)$ & $0.89(0.85-0.93)$ & $0.90(0.85-0.95)$ \\
\hline $\begin{array}{l}\text { Use of dyslipidemia drugs } \\
\text { at baseline }\end{array}$ & $0.87(0.83-0.92)$ & $0.92(0.88-0.97)$ & $0.96(0.91-1.02)$ \\
\hline $\begin{array}{l}\text { Use of antithrombotics } \\
\text { at baseline }\end{array}$ & $1.09(1.02-1.15)$ & $1.05(0.99-1.11)$ & $1.01(0.94-1.07)$ \\
\hline
\end{tabular}

Abbreviations: OR, odds ratio; CI, confidence interval.

\section{Discussion}

In this study, the analyses of claims data from 226,738 community-dwelling elderly individuals in Niigata prefecture in Japan revealed a significantly higher risk of new dementia diagnoses among those with comorbidities of cerebrovascular disease, as well as those who are prescribed antidepressants, antipsychotics, or hypnotics, especially in the 75-79-year age group. According to the type of dementia, cerebrovascular disease and antipsychotic use were common significant predictors for Alzheimer's disease and vascular dementia, with a stronger association observed with vascular dementia. Depression/antidepressant use was also a common significant predictor for Alzheimer's disease and vascular dementia. These results suggest the usefulness of administrative claims data for identifying new dementia diagnoses.

The present study found neurological or psychiatric factors to be the most positively associated predictors across dementia sub-types and age groups, with significant associations observed for depression and cerebrovascular disease. Cerebrovascular disease has been shown to increase the risk of developing dementia [12]. In most cases, dementia has a mixed pathology of both vascular and neurodegenerative factors $[13,14]$. In addition, cerebrovascular disease can promote Alzheimer's disease and vice versa, resulting in a reciprocal interaction amplifying the respective pathogenic effects $[15,16]$. Thus, controlling the established cerebrovascular risk factors can be beneficial as it reduces the risk of subsequent dementia [17]. We found that depression was a predictor for dementia, which is consistent with previous reports [6,18]. However, whether depressive symptoms are an early sign of dementia or an independent risk factor for dementia remains unclear, and the present findings of 4-year follow-up do not provide insight as to whether depressive symptoms preceded the diagnosis of dementia. A recent study has suggested that depression is potentially preventable and may be a modifiable risk factor of subsequent dementia [19]. Longer follow-up is necessary to clarify these aspects.

With regard to cardiovascular risk factors, current use of antihypertensive drugs and dyslipidemia drugs negatively predicted subsequent dementia diagnosis among people aged $\geq 75$ years. This finding is consistent with previous reports [20]. There is sufficiently strong evidence that the management of cardiovascular risk factors is a promising approach to prevent dementia [3]. Previous longitudinal studies have shown that the timing of exposure to vascular predictors may be critical. Midlife-but not late-life-hypertension 
is reportedly associated with a higher risk of cognitive decline [21], and this risk may be reduced by treatment of hypertension [6,22]. We found similar results, which reinforced the hypothesis that the association between cardiovascular risk factors and dementia differed by the age of the target population.

Consistent with results from previous studies $[6,23,24]$, we found that diabetes mellitus was a significant predictor for developing dementia. However, this association was only observed in individuals aged 75-79 years (Table 4), suggesting that predictors of dementia may vary by age. The present analyses of administrative claim data did not clarify whether sustained hyperglycemia, long-term use of anti-diabetic drugs, or repeated drug-induced hypoglycemia are primary causes leading to the development of dementia. Further elucidation of the causality and appropriate clinical measures will require longitudinal and detailed clinical data, including those on the status of medication and blood glucose levels.

A meta-analysis of observational studies using routine clinical data from Western countries showed that neuropsychiatric symptoms including depression, anxiety, antipsychotic use, and history of stroke were positively associated with all dementia [25]. On the other hand, among a few studies using only administrative claim data, one exploratory casecontrol study reported that schizophrenia, depression, and stroke increased subsequent dementia risk [26]. The present study using claims data identified dementia predictors that were consistent with those reported previously. Routinely collected clinical data, including healthcare claims, will help in estimating population burden of dementia for policy making, as well as for practical decision making.

The strength of this study is the use of comprehensive prefecture-level claims data of people over 75 years of age. These data cover the total population, including people who live in institutions such as assisted living or nursing homes, which enhanced our study generalizability. On the other hand, this study had several limitations. First, administrative claims contain diagnoses that are coded for the reimbursement of medical services. In other words, dementia might be underreported because only diagnoses related to an actual treatment are recorded; therefore, those with dementia who did not consult a doctor could not be identified. To address this issue, we adopted conservative criteria for the outcome definition, reducing the possibility of overestimation and increasing the validity of the outcome. Second, we did not completely differentiate between dementia subtypes, as is often the case with studies using claims data [11]. Vascular dementia might be underreported due to the lack of a definite therapeutic agent, and might also be misclassified as unspecified dementia. Third, these data were only from fiscal years 2012 and 2016, while those from fiscal years 2013-2015 were not available due to administrative reasons. Therefore, individuals who were newly diagnosed with dementia between 2013-2015 and subsequently died or emigrated before 2016 could not be assessed. Fourth, there may be residual confounding factors. We adjusted for age, sex, cardiovascular risk factors, and neurological or psychiatric risk factors, but other predictors of dementia (e.g., educational level, ApoE4 allele carrier status) could not be integrated into the analysis because our dataset lacked detailed sociodemographic parameters and genetic information. Fifth, the lack of clinical data such as blood pressure, blood glucose, and blood lipid levels might have limited the validity of causal inference of hypertension, diabetes mellitus, dyslipidemia, and medication for these diseases for developing subsequent dementia.

\section{Conclusions}

The analyses of administrative claims data revealed that comorbidities of cerebrovascular disease and depression, antipsychotic use, and hypnotic use are predictors of new dementia diagnoses in the general population of people aged $\geq 75$ years.

Supplementary Materials: The following materials are available online at https: / www.mdpi.com/ 1660-4601/18/2/629/s1: Table S1. Multivariable logistic regression analyses of predictors for new diagnosis of Alzheimer's disease; Table S2. Multivariable logistic regression analyses of predictors for new diagnosis of vascular dementia. 
Author Contributions: Conceptualization, Y.N.; methodology, Y.N., Y.T., S.T., and T.N.; formal analysis, Y.N.; writing-original draft preparation, Y.N.; writing—review and editing, Y.T., S.T., and T.N.; supervision, T.N.; project administration, Y.N. All authors have read and agreed to the published version of the manuscript.

Funding: This study was supported by donations to the Department of Health Informatics, Kyoto University School of Public Health from the JMDC and JSPS KAKENHI Grant Number 20H01594.

Institutional Review Board Statement: The study was conducted according to the guidelines of the Declaration of Helsinki, and approved by the Ethics Committee of the Kyoto University Graduate School and Faculty of Medicine (approval number R1767, 23 October 2018).

Informed Consent Statement: Patient consent was waived because this study involved routinely collected claims data that were de-identified before provision and anonymously managed at all stages, including data cleaning and statistical analysis.

Data Availability Statement: Restrictions apply to the availability of these data. Data was obtained from the Niigata wide area union of the medical care system for the elderly in Japan and are not publicly available. Data are however available from the authors upon reasonable request and with the permission of the Niigata wide area union.

Acknowledgments: We thank JMDC for their data processing of the medical claims. We thank the Niigata wide area union for providing us with the data.

Conflicts of Interest: The authors declare no conflict of interest.

\section{References}

1. Patterson, C. World Alzheimer Report 2018; Alzheimer's Disease International: London, UK, 2018; Available online: https: / / www.alz.co.uk/research/world-report-2018 (accessed on 29 October 2020).

2. Ministry of Health, Labour and Welfare. Long-Term Care Insurance System of Japan. Available online: https:/ / www.mhlw.go. jp/english/policy/care-welfare/care-welfare-elderly/ (accessed on 29 October 2020).

3. Ohara, T.; Hata, J.; Yoshida, D.; Mukai, N.; Nagata, M.; Iwaki, T.; Kitazono, T.; Kanba, S.; Kiyohara, Y.; Ninomiya, T. Trends in dementia prevalence, incidence, and survival rate in a japanese community. Neurology 2017, 88, 1925-1932. [CrossRef] [PubMed]

4. Baumgart, M.; Snyder, H.M.; Carrillo, M.C.; Fazio, S.; Kim, H.; Johns, H. Summary of the evidence on modifiable risk factors for cognitive decline and dementia: A population-based perspective. Alzheimers Dement. 2015, 11, 718-726. [CrossRef] [PubMed]

5. Norton, S.; Matthews, F.E.; Barnes, D.E.; Yaffe, K.; Brayne, C. Potential for primary prevention of alzheimer's disease: An analysis of population-based data. Lancet Neurol. 2014, 13, 788-794. [CrossRef]

6. Livingston, G.; Huntley, J.; Sommerlad, A.; Ames, D.; Ballard, C.; Banerjee, S.; Brayne, C.; Burns, A.; Cohen-Mansfield, J.; Cooper, C.; et al. Dementia prevention, intervention, and care: 2020 report of the Lancet Commission. Lancet 2020, 396, 413-446. [CrossRef]

7. Barnes, D.E.; Yaffe, K. The projected effect of risk factor reduction on alzheimer's disease prevalence. Lancet Neurol. 2011, 10, 819-828. [CrossRef]

8. Stephan, B.C.; Tang, E.; Muniz-Terrera, G. Composite risk scores for predicting dementia. Curr. Opin. Psychiatry 2016, 29, 174-180. [CrossRef]

9. Tang, E.Y.; Harrison, S.L.; Errington, L.; Gordon, M.F.; Visser, P.J.; Novak, G.; Dufouil, C.; Brayne, C.; Robinson, L.; Launer, L.J.; et al. Current developments in dementia risk prediction modelling: An updated systematic review. PLoS ONE 2015, 10, e0136181. [CrossRef]

10. Walters, K.; Hardoon, S.; Petersen, I.; Iliffe, S.; Omar, R.Z.; Nazareth, I.; Rait, G. Predicting dementia risk in primary care: Development and validation of the dementia risk score using routinely collected data. BMC Med. 2016, 14, 6. [CrossRef]

11. Hessler, J.B.; Ander, K.H.; Brönner, M.; Etgen, T.; Förstl, H.; Poppert, H.; Sander, D.; Bickel, H. Predicting dementia in primary care patients with a cardiovascular health metric: A prospective population-based study. BMC Neurol. 2016, 16, 116. [CrossRef]

12. Kuźma, E.; Lourida, I.; Moore, S.F.; Levine, D.A.; Ukoumunne, O.C.; Llewellyn, D.J. Stroke and dementia risk: A systematic review and meta-analysis. Alzheimers Dement. 2018, 14, 1416-1426. [CrossRef]

13. Iadecola, C. The pathobiology of vascular dementia. Neuron 2013, 80, 844-866. [CrossRef] [PubMed]

14. Jellinger, K.A. Pathology and pathogenesis of vascular cognitive impairment-a critical update. Front. Aging Neurosci. 2013, 5, 17. [CrossRef] [PubMed]

15. Gorelick, P.B.; Scuteri, A.; Black, S.E.; Decarli, C.; Greenberg, S.M.; Iadecola, C.; Launer, L.J.; Laurent, S.; Lopez, O.L.; Nyenhuis, D.; et al. Vascular contributions to cognitive impairment and dementia: A statement for healthcare professionals from the american heart association/american stroke association. Stroke 2011, 42, 2672-2713. [CrossRef] [PubMed]

16. Chui, H.C.; Zheng, L.; Reed, B.R.; Vinters, H.V.; Mack, W.J. Vascular risk factors and alzheimer's disease: Are these risk factors for plaques and tangles or for concomitant vascular pathology that increases the likelihood of dementia? An evidence-based review. Alzheimers Res. Ther. 2012, 4, 1. [CrossRef] [PubMed] 
17. Toledo, J.B.; Arnold, S.E.; Raible, K.; Brettschneider, J.; Xie, S.X.; Grossman, M.; Monsell, S.E.; Kukull, W.A.; Trojanowski, J.Q. Contribution of cerebrovascular disease in autopsy confirmed neurodegenerative disease cases in the national alzheimer's coordinating centre. Brain 2013, 136, 2697-2706. [CrossRef] [PubMed]

18. Diniz, B.S.; Butters, M.A.; Albert, S.M.; Dew, M.A.; Reynolds, C.F., 3rd. Late-life depression and risk of vascular dementia and alzheimer's disease: Systematic review and meta-analysis of community-based cohort studies. Br. J. Psychiatry 2013, 202, 329-335. [CrossRef]

19. Reynolds, C.F., 3rd; Cuijpers, P.; Patel, V.; Cohen, A.; Dias, A.; Chowdhary, N.; Okereke, O.I.; Dew, M.A.; Anderson, S.J.; Mazumdar, S.; et al. Early intervention to reduce the global health and economic burden of major depression in older adults. Annu. Rev. Public Health 2012, 33, 123-135. [CrossRef]

20. Solomon, A.; Mangialasche, F.; Richard, E.; Andrieu, S.; Bennett, D.A.; Breteler, M.; Fratiglioni, L.; Hooshmand, B.; Khachaturian, A.S.; Schneider, L.S.; et al. Advances in the prevention of alzheimer's disease and dementia. J. Intern. Med. 2014, 275, 229-250. [CrossRef]

21. Gottesman, R.F.; Schneider, A.L.; Albert, M.; Alonso, A.; Bandeen-Roche, K.; Coker, L.; Coresh, J.; Knopman, D.; Power, M.C.; Rawlings, A.; et al. Midlife hypertension and 20-year cognitive change: The atherosclerosis risk in communities neurocognitive study. JAMA Neurol. 2014, 71, 1218-1227. [CrossRef]

22. Rouch, L.; Cestac, P.; Hanon, O.; Cool, C.; Helmer, C.; Bouhanick, B.; Chamontin, B.; Dartigues, J.F.; Vellas, B.; Andrieu, S. Antihypertensive drugs, prevention of cognitive decline and dementia: A systematic review of observational studies, randomized controlled trials and meta-analyses, with discussion of potential mechanisms. CNS Drugs 2015, 29, 113-130. [CrossRef]

23. Ohara, T.; Doi, Y.; Ninomiya, T.; Hirakawa, Y.; Hata, J.; Iwaki, T.; Kanba, S.; Kiyohara, Y. Glucose tolerance status and risk of dementia in the community: The hisayama study. Neurology 2011, 77, 1126-1134. [CrossRef] [PubMed]

24. Chatterjee, S.; Peters, S.A.; Woodward, M.; Arango, S.M.; Batty, G.D.; Beckett, N.; Beiser, A.; Borenstein, A.R.; Crane, P.K.; Haan, M.; et al. Type 2 diabetes as a risk factor for dementia in women compared with men: A pooled analysis of 2.3 million people comprising more than 100,000 cases of dementia. Diabetes Care 2016, 39, 300-307. [CrossRef] [PubMed]

25. Ford, E.; Greenslade, N.; Paudyal, P.; Bremner, S.; Smith, H.E.; Banerjee, S.; Sadhwani, S.; Rooney, P.; Oliver, S.; Cassell, J. Predicting dementia from primary care records: A systematic review and meta-analysis. PLoS ONE 2018, 13, e0194735. [CrossRef] [PubMed]

26. Albrecht, J.S.; Hanna, M.; Kim, D.; Perfetto, E.M. Predicting Diagnosis of Alzheimer's Disease and Related Dementias Using Administrative Claims. J. Manag. Care Spec. Pharm. 2018, 24, 1138-1145. [CrossRef] [PubMed] 